\title{
A Rare Case of Triple Malignancy: Non Hodgkin Lymphoma, Carcinoma of Breast and Squamous Cell Carcinoma of Tongue in a Single Patient
}

\author{
*M E Haque ${ }^{1}, \mathrm{~S} \mathrm{Karim}^{2}$, M M R Siddiqui ${ }^{3}, \mathrm{M} \mathrm{kareem}^{4}$, A Khatun ${ }^{5}$
}

\begin{abstract}
We are reporting a case of having triple malignancy including Non Hodgkin Lymphoma (NHL), Carcinoma breast, Squamous cell carcinoma (SCC) of the tongue. Triple malignancy is a very rare occurrence. Cancer patient are at increased risk of developing a subsequent primary tumour. Prevalence of multiple primary malignancies is slowly increasing due to prolonged survival of cancer patients with advances in diagnostic and therapeutic modalities. The reasons may be environmental modifications, genetic predisposition or therapy-induced. People who have had non-Hodgkin lymphoma (NHL) can get any type of second cancer, but they have an increased risk of certain cancers, including carcinoma lung, skin, tongue, bladder, colon, kidney etc. Radiation therapy to the chest increases the risk of breast cancer.
\end{abstract}

Key Words: Triple primary malignancy of a patient including NHL, carcinoma breast and carcinoma tongue

\section{Introduction}

Multiple primary cancers are usually defined as primary malignant tumors of different histological origins in one person. Prevalence of multiple primary malignancies is slowly increasing due to prolonged survival of cancer patients with advances in diagnostic and therapeutic modalities. ${ }^{1}$ The reasons may be environmental modifications, genetic predisposition or therapy induced. ${ }^{1}$ The number of cancer survivors is growing by $2 \%$ each year with advances in diagnostic modalities, treatment and supportive care. ${ }^{2}$ An individual may develop MPM in lifetime due to genetic predisposition, environmental exposure to carcinogens, immunodeficiency or as a serious complication of chemotherapy or radiotherapy received for first primary malignancy. ${ }^{3}$ National Cancer Institute's Surveillance, Epidemiology, and End Results Program reported that about one in six cancer patients develop second malignant neoplasm in their further lifetime. ${ }^{3}$
Recently, there has been an increase in the number of patients diagnosed with multiple primary cancers. This trend can be attributed to improved diagnostic techniques, prolonged lifespan and the increased incidence of long-term survival of patients with malignancy. Most multiple primary cancers are double primary cancers. ${ }^{4,5}$

Definitions and classifications for multiple primary cancers and multi-centric cancers, proposed by Moertel way back in 1977 hold true even today. 5,6 Accordingly, group I includes, multiple primary cancers occurring in organs with the same histology, group II includes multiple primary cancers that originate from different tissues and group III consists of cancers from different tissues and organs that concurrently exist with group I cancers, and they form multiple primary cancer of three or more cancers. Group I is further subdivided into group A, which includes cancers that occur in the same tissue and organ, group B, which includes cancers that are

$1 *$ Prof. Dr. Md. Ehteshamul Haque, Professor \& Head, Dept. of Oncology. AKMMC

${ }^{2}$ Dr. Shahnaz Karim, Associate Professor, Dept. of Transfusion Medicine. DMCH

${ }^{3} \mathrm{Dr}$. Md. Mahmudur Rahman Siddiqui, Associate Professor, Dept. of Medicine, AKMMC

${ }^{4}$ Dr. Mayesha Kareem, Medical Officer, Dept. of Medicine. AKMMC

${ }^{5}$ Dr. Amena Khatun, Medical Officer, Dept. of Oncology. AKMMC

*Corresponding Author

Date of submission: 10.09.2017, Date of acceptance: 12.11.2017 
from the same tissue and different organs, and group $\mathrm{C}$, which includes cancers that occur in bilateral organs. Multiple primary cancers are again classified as synchronous and metachronous. Those malignancies that are observed at the same time or within 6 months are termed as synchronous multiple primary cancers, and those cancers that develop at more than a 6-month interval are termed as metachronous multiple primary cancers. ${ }^{7}$ On the other hand, many studies have defined 1 year as the dividing time of these two types of multiple cancers. ${ }^{8}$

\section{Case Report}

A 71-year-old female, nonobese, nonsmoker, nonalcoholic with no family history of malignancy hailing from 40, presented with respiratory distress for 2 weeks. Had the history of non-Hodgkin lymphoma on 2007, which was diagnosed by PETCT \& confirmed by lt cervical lymph node biopsy \&treated with chemotherapy; RCHOP -14 (cyclophosphamide + adriamycin + vincristine + prednisolone + rituximab) 2 weekly for six cycles started on $18 / 07 / 07$. The treatment period was uneventful \& lymphoma was subsided which was confirmed by PET-CT. In 2010, she developed the lump on left breast $\&$ her mammography revealed the malignancy. Frozen section biopsy was done per operatively shows invasive carcinoma of the left breast with axillary lymph node metastasis. For that, she was treated with surgery. Mastectomy \& axillary lymph node dissection followed by oral chemotherapy for 5 years. She was on regular follow up for that \& the treatment response was good without developing any complication. In 2017, during regular follow up her tongue was coated $\&$ rough.

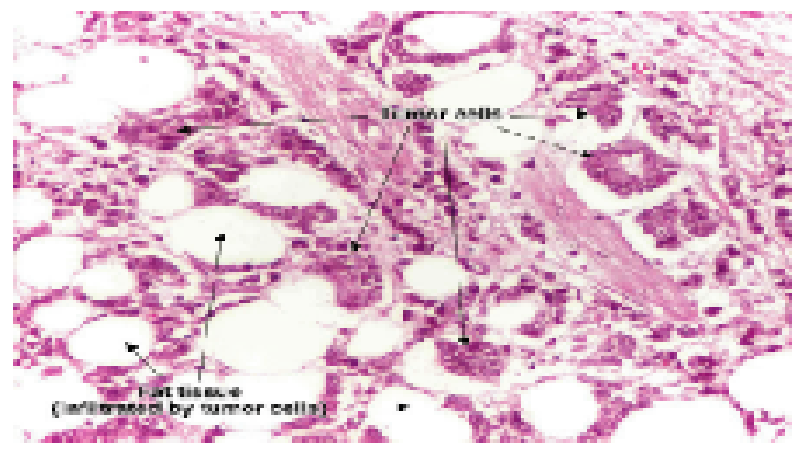

Fig.-1: Infiltrating ductal cell carcinoma of breast
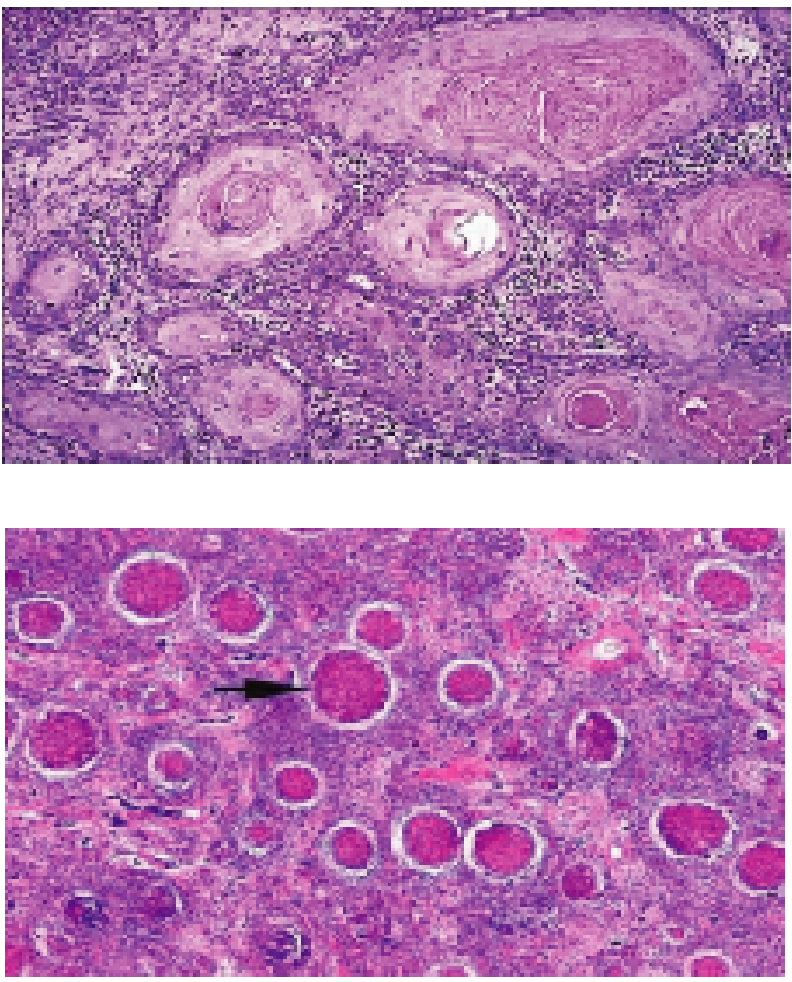

Fig.-3: Large follicular B-cell lymphoma

The biopsy was done, which shows leukoplakia in the tongue. In 2017, the incisional biopsy of tongue shows- Squamous cell carcinoma of the tongue. After that she had undergone--Partial glossectomy with reconstruction with left modified radical neck dissection followed by chemotherapy with cisplatin weekly for five cycles started on 26/04/17 \& Radiotherapy for thirty-five cycle (25/04/17-14/06/17). For chemotherapy, she developed neutropenic sepsis \& treated with the proper antibiotic. For that, she was on regular follow up \& chemotherapy was stopped from developing a complication. After that patient's condition deteriorating\& admitted in hospital on-/08/17 with a headache and severe respiratory distress for last 2 weeks due to laryngeal edema. As patient's present condition only permits her for palliative treatment, so permanent tracheostomy was done on $19 / 08 / 17$ to prevent sudden airway obstruction \& for her better well-being. She is a case of triple malignancy-- nonHodgkin (follicular large B cell lymphoma Grade 3, stage 4), Ca left breast with squamous cell carcinoma of tongue with HTN, newly diagnosed DM. now her disease progression is successfully managed by current treatment. 


\section{Discussion}

Though multiple primary cancers are not common, yet it is believed that the incidence is increasing. Since in patients with multiple cancers, the focus is mainly on the primary disease, there is a higher likelihood of missing incidental co-existence of another primary malignant lesion. Therefore, it is important to make an early diagnosis and administer prompt therapy in case of multiple cancers. ${ }^{6}$ Cancers survivors had a $14 \%$ higher risk of developing a new malignancy that would have been expected in the general population according to the cancer registries in the National Cancer Institute. Woman had a slightly higher relative risk than men for multiple primary malignancies, and the most implicated sites were colon, breast, lung, and melanoma of the skin. ${ }^{19}$

Multiple primary malignant neoplasms can occur at any age. However, from the reviewed series, the patients with MPMN tend to be older than those with a single primary malignant neoplasm. The mechanisms explaining the association between cancer and aging include:

- Time length of carcinogenesis; the longer a person lives the more likely it is that carcinogenesis will be completed and cancer will develop. ${ }^{10}$

- Molecular changes of age; older tissues are susceptible to environmental carcinogenesis and undergo molecular changes similar to carcinogenesis. 10

- Changes in the environment; aging is associated with molecular changes in DNA signaling and body environment that may favor the development of cancer. ${ }^{9}$

The global cancer burden according to IARC in 2012 was 14.1 million new cases and 8.2 million cancer deaths and is estimated to increase by 2030 to 21.7 million new cases and 13 million deaths. ${ }^{16}$ With this increase alone, the number of patients with multiple primaries will grow significantly.

According to classification stated by Moertel way back in 1977, this patient has metachronous multiple primary malignancies as her first primary malignancy was NHL diagnosed by biopsy from cervical lymph node in 2007, second primary malignancy was carcinoma left breast which was suspected by mammography and was confirmed by frozen section biopsy in 2010, and her last primary malignancy was carcinoma tongue which was diagnosed by incision biopsy in 2017. The interval between NHL to carcinoma breast and carcinoma breast to carcinoma tongue both periods was $>6$ month. With better treatment, life expectancy of NHL patients has been improving 17 and hence the risk of iatrogenic malignancy is becoming an important concern in long-term survivors. ${ }^{18}$

After the diagnosis of NHL this patient was treated with R-CHOP regimen, whole treatment period was uneventful and NHL was subsided which was confirmed by PET-CT scan.

According to American cancer society Lymphoma survivors should also follow the American Cancer Society recommendations for the early detection of cancer, such as those for colorectal, lung, and breast cancer. To help maintain good health, lymphoma survivors should also: Avoid tobacco product, Get to and stay at a healthy weight, Stay physically active, Eat a healthy diet, with an emphasis on plant foods, Limit alcohol to no more than 1 drink per day for women or 2 per day for men, These steps may also lower the risk of some other health problems.

In patients with breast cancer, the incidence of multiple primaries has been reported in the range of $4.1 \%$ (Kim et al 108 patients, 45.9 months follow-up, guideline not available) to $16.4 \%$ (Weir et al 301963 patients, max. 10 years follow-up, SEER, in IACR only $10.4 \%) 12,13,14$. The median time to a second malignancy was between 5 and 8 years. ${ }^{14,15}$ This patient develope third primary malignancy (carcinoma tongue) after 7-year carcinoma. After the diagnosis of carcinoma breast this patient undergone operative management followed by chemotherapy and treatment show excellent response and was under regular checkup, that's why carcinoma tongue was diagnosed in stage-1. After the diagnosis of carcinoma tongue patient received 35 fractions of radiotherapy. Maintaining of general health condition is very important during treatment of any cancer as any type of chemotherapeutic agent or radiotherapy are very toxic to normal tissue. 
Very often patient develop neutropenia after treatment which can lead to neutropenic sepsis. That is why granulocyte colony stimulating factor used to given on injectable (subcutaneous) form. This patient also develops neutropenia after receiving of radiotherapy which leads to neutropenic sepsis and her general condition severely deteriorated. And finally, she treated only by palliative care.

\section{Interest of conflict: None}

\section{Conclusion}

Because of advanced treatment protocol life expectancy in cancer patient increasing greatly but by this time risk of developing multiple primary carcinomas is also increasing. Regular follow-up of the cancer survivor must detect Multiple Primary Carcinoma in the early stage as well as recurrence of carcinoma.

\section{References}

1. Cercato M. C., Colella E., Ferraresi V., et al. Report of two cases of quintuple primary malignancies and review of the literature. Anticancer Research. 2008; 28: 2953-2958.

2. Wood M. E., Vogel V., Ng A., Foxhall L., et al. Second malignant neoplasms: assessment and strategies for risk reduction. Journal of Clinical Orthodontics: JCO. 2012; 30: 3734-3745.

3. American cancer society report 2015.

4. Noh SK, Yoon JY, Ryoo UN, et al. A case report of quadruple cancer in a single patient including the breast, rectum, ovary, and endometrium. J Gynecol Oncol. 2008; 19: 265-9.

5. Iioka $\mathrm{Y}$, Tsuchida $\mathrm{A}$, Okubo $\mathrm{K}$, et al. Metachronous triple cancers of the sigmoid colon, stomach, and esophagus: Report of a case. Surg Today. 2000; 30: 368-71.

6. Lee JS, Moon W, Park SJ, et al. Triple synchronous primary cancers of rectum, thyroid, and uterine cervix detected during the workup for hematochezia. Intern Med. 2010; 49:1745-7.

7. Moertel CG. Multiple primary malignant neoplasms: Historical perspectives. Cancer. 1977; 40: 1786-92.
8. Moertel CG, Dockerty MB, Baggenstoss AH. Multiple primary malignant neoplasms. I. Introduction and presentation of data. Cancer. 1961; 14: 221-30.

9. Hu NC, Hsieh SC, Chen TJ, Chang JY. Multiple primary malignancies including colon, stomach, lung, breast, and liver cancer: a case report and literature review. Chin Med J (Engl) 2004; 122: 3091-3093.

10. archives of surgical oncology

11. Ferlay J, Steliarova-Foucher E ,Lortet-Tieulent $\mathrm{J}$, et al Cancer incidence and mortality patterns in Europe: estimates for 40 countries in 2012. Eur J Cancer 2013; 49: 1374-403.

12. Weir HK, Johnson CJ ,Thompson TD The effect of multiple primary rules on population-based cancer survival. Cancer Causes Control 2013; 24:1231-42.

13. Amer MH Multiple neoplasms, single primaries, and patient survival. Cancer Manag Res 2014; 6:119-34

14. KimJYSong HSMetachronous double primary cancer after treatment of breast cancer. Cancer Res Treat 2015; 47: 64-71.

15. RicceriF, FasanelliF Giraudo MT, et alRisk of second primary malignancies in women with breast cancer: results from the European prospective investigation into cancer and nutrition (EPIC). Int J Cancer 2015; 137: 940-8.

16. Ferreti Sea. Airtum cancer registration handbook, 2009.

17. National Cancer Institute: SEER Cancer Statistics Review, 1975-2001. http:// seer. cancer. gov/csr/1975_2001

18. Swerdlow AJ, Van Leeuwen FE: Late effects after treatment for Hodgkin lymphoma, in Dembo AJ, Linch DC, Lowenberg B (eds): Textbook of Malignant Hematology. Abingdon, United Kingdom, Taylor \& Francis, pp 753, 2005-768

19. R.E. Curtis, D.M. Freedman, E. Ron, et al. Fraumeni New Malignancies Among Cancer Survivors: SEER Cancer Registries, 1973-2000 National Cancer Institute, Bethesda, MD (2006) NIH Publ. No. 05-5302 